\title{
LES PRATIQUES TOURISTIQUES DES TOURISTES FRANÇAIS EN CHINE ${ }^{1}$
}

\author{
Lu LI
}

\begin{abstract}
Résumé
La Chine avec sa forte altérité attire l'attention des Français et génère un flux touristique important. Notre étude vise à mettre en avant leurs pratiques touristiques en Chine, surtout à partir des lieux visités ainsi que leurs activités touristiques sur place. À travers une analyse des produits touristiques, des entretiens avec des professionnels ainsi qu'une enquête auprès des touristes français, nous avons découvert que les touristes français fréquentent toujours des hauts lieux touristiques, souvent dans des villes emblématiques dans l'est de la Chine. La découverte culturelle domine généralement dans leur visite avec des activités étroitement liées à la recherche de l'altérité chinoise. Le tourisme des touristes français en Chine ainsi que son évolution sont le résultat de l'intervention commune de l'État chinois et du marché touristique.
\end{abstract}

Mots-clés

pratiques touristiques, touristes français, Chine, produits touristiques

\begin{abstract}
With its strong alterity, China attracts French tourists'attention and has generated an important touristic flux. Our study aims at revealing their tourism in China, especially regarding the places visited by French tourists and their main touristic activities. By analyzing touristic products, interviewing professionals, as well as investigating French tourists, we have found that French tourists often visit classical highland, usually in symbolic cities in eastern China. Then, the culture discovery dominates their visits, which is closely related to the pursuit of Chinese alterity. The tourism of French tourists in China is the result of the joint intervention of Chinese government and tourism market.
\end{abstract}

Keywords

touristic practices, French tourists, China, touristic products

\section{INTRODUCTION}

Pendant une quarantaine d'années, le nombre d'arrivées de visiteurs français en Chine a connu une croissance considérable, allant de 15000 visiteurs français en 1980 à 499600 en $2018^{2}$. Selon l'Office national du Tourisme de Chine à Paris, 45 agences françaises intègrent la Chine dans leurs destinations, couvrant presque tous les voyagistes français. Avec une civilisation datant de plusieurs millénaires, un territoire vaste d'une grande diversité et un peuple très différent, la Chine diffère des destinations traditionnelles des Français, qui sont analysées par de nombreuses études qui montrent qu'ils sont passionnés par le balnéaire et la campagne, avec une préférence pour le tourisme à l'intérieur de la France. Face à une destination si dépaysante et unique comme la Chine, comment les touristes français y font-ils du tourisme?

\section{LA PRATIQUE TOURISTIQUE : UNE AP- PROCHE SUR LE TOURISME}

Des chercheurs proposent une lecture du tourisme à partir des touristes (Mondou et Violier, 2009) et ils ont essayé de comprendre leurs comportements au travers de multiples aspects : la motivation, le processus de la prise de décision, la satisfaction, les impacts des expériences touristiques... Ce sont les principales pistes de recherche sur les comportements du touriste. Nous pouvons remarquer que la recherche se concentre en grande partie sur la phase avant le départ et celle après le retour. Cependant, la phase " du milieu », celle qui montre ce que fait le touriste et comment le voyage se déroule, mériterait plus d'attention.

La notion de la pratique est bien courante dans le domaine de la culture et des loisirs, son implica- 
tion dans l'étude du tourisme a été proposée tout d'abord par Pascal Cuvelier (1998). Pour lui, le concept de la pratique englobe tout un ensemble de processus et de conditions de l'univers touristique, et il permettrait d'insister sur les différentes dimensions constitutives de l'épaisseur du produit touristique, d'intégrer le degré de participation du touriste (Cuvelier, 1998). La pratique touristique constitue la clé qui sert à lier les lieux touristiques et les touristes, puisqu'elle met l'accent sur ce que fait un touriste pendant son voyage, qui combine « la mise en acte de ses intentions et des réponses qu'il apporte aux suggestions du lieu » (L'Équipe MIT, 2002). Ainsi, pour mettre en oeuvre un projet touristique, le touriste choisit un/des lieu(x) conformément à son intention pour ensuite le/les pratiquer. Par conséquent, les pratiques touristiques, catégorisées principalement en découverte, repos, jeu, shopping, sociabilité (L'Équipe MIT, 2002, 2005, 2011), correspondent à la qualité du lieu tout en répondant au projet touristique du touriste. L'étude sur les pratiques des touristes permettent de mieux comprendre le rapport entre le touriste et le lieu ainsi que le système du tourisme.

Différents auteurs ont déjà relevé des différences entre les pratiques touristiques chinoises et celles des étrangers (Nyiri, 2006 ; Taunay, 2009 ; Taunay et Violier, 2015). Si les distinctions de pratiques touristiques sont repérées entre les touristes chinois et les touristes internationaux sur le territoire chinois, peu d'études ont été faites en ce qui concerne les touristes d'un pays spécifique pour approfondir l'analyse de leurs pratiques qui varieraient d'un pays à un autre étant donné les différences culturelles, sauf quelques travaux sur, par exemple, la segmentation marketing des touristes américains pour la Chine (Qu et al., 2018), la perception des touristes japonais sur la promotion touristique de la région autonome du Xinjiang Uighur (Axiamujiang et al., 2016). Ainsi, notre recherche vise à mettre en lumière le tourisme des touristes français en Chine, à travers leurs pratiques touristiques qui se traduisent en particulier par deux questions : où vont-ils et que font-ils en Chine ?

\section{MÉTHODE}

L'approche institutionnelle confondant différentes mobilités sous une appellation globale du « tourisme » produirait des statistiques avec peu de sens (Taunay et Violier, 2015). Des chercheurs
(Santos, 1998 ; Violier, 2011 ; Taunay et Violier, 2015) ont proposé une analyse des catalogues des tour-opérateurs (TO), car les professionnels comme ces derniers possèdent de réelles connaissances sur la destination pour concevoir leurs produits touristiques (Tomigová et al., 2016). Il s'agit d'une analyse fréquentielle des lieux cités dans les circuits et séjours ainsi que les activités sur les lieux proposés par les TO. Cette méthode a été appliquée par Violier (2011) et Taunay et Violier (2015) dans leurs études sur les lieux et les pratiques des touristes internationaux et des touristes chinois visitant la Chine, l'analyse des catalogues des TO étant pertinente dans le cadre de longs déplacements quand le recours à un intermédiaire est plus élevé (Mondou et Violier, 2009). Pourtant, il existe des biais dans cette méthode (Taunay et Violier, 2015) : les prestations sur les catalogues ne relèvent que des propositions et des excursions réelles ne sont pas divulguées ; les touristes qui ne font pas appel aux TO ne sont pas inclus. De ce fait, nous choisissons de travailler sur les catalogues des sites Internet officiels de quelques grands TO proposant la Chine. La rubrique « avis » permet aux clients d'évaluer les prestations et de commenter leur voyage en Chine, le nombre d'avis laissés sur différents itinéraires révèlent en quelque sorte les best-sellers qui décryptent leurs préférences pour les lieux touristiques en Chine. Ensuite, des entretiens avec des agences de voyages réceptives permettent de vérifier les résultats et de présenter l'évolution de la fréquentation des lieux touristiques auprès des touristes français en Chine pendant 40 ans. Enfin, une enquête par questionnaire est menée auprès de touristes français en Chine pour dégager les lieux les plus fréquentés ainsi que les activités les plus pratiquées. Ces analyses nous donnent des informations sur les véritables pratiques des touristes français en Chine, y compris les touristes plus autonomes.

Le choix du lieu d'enquête est exigeant. Vu l'immensité du territoire et la dispersion des lieux touristiques, il n'est en effet pas possible d'être exhaustif pour tous les lieux fréquentés par les touristes français en Chine, même pour les grandes métropoles comme Pékin, Shanghaï, et Canton, où il est difficile de constituer des échantillons car les touristes sont constamment en déplacement et ces villes sont trop grandes pour les repérer. Par contre, Yangshuo, un district de la ville touristique de Guilin, nous semble un lieu parfait pour l'enquête 
parce qu'il accueille des visiteurs internationaux qui se concentrent surtout dans la rue de l'Ouest, centre du district. Le plus important, c'est son attrait et sa réputation en tant que lieu touristique incontournable depuis le début du tourisme récepteur en Chine (Bai, 2005), des circuits classiques ou des voyages en profondeur l'incluant souvent dans leurs itinéraires car il représente l'idéal de la Chine immobile et éternelle avec ses campagnes, rizières et montagnes, peuplées de paysans en chapeau de paille. Malgré des limites, comme sa situation dans le sud, qui pourraient affecter la représentativité de l'échantillon, Yangshuo nous paraissait le meilleur choix comme lieu d'enquête. Ce choix nous a permis d'interroger environ 700 touristes français pour obtenir 515 questionnaires valides.

\section{LES PRINCIPAUX ITINÉRAIRES TOURISTIQUES POUR LES TOURISTES FRANÇAIS EN CHINE}

\section{A. Analyse des trois grands TO français}

À partir des catalogues présentés sur les sites Internet officiels en 2019 des trois grands tour-opérateurs proposant la Chine, soient Asia, La Maison de la Chine et Voyageurs du Monde, nous avons obtenu les produits les plus achetés par les touristes français selon le nombre d'avis des clients : « Chine Intimiste », « Chine Essentielle » et « Dragons de Chine » d'Asia, «Toute la Chine » et « Grands sites de la Chine classique » de La Maison de la Chine, «Premiers pas en Chine » et « La Chine avec des enfants » de Voyageurs du Monde. Il s'agit des circuits classiques plus ou moins similaires avec les choix des lieux les plus typiques et les plus véhiculés par les médias. Comme présenté dans la Carte 1, le voyage commence par Pékin, desservi par le vol direct avec Paris, les touristes vont souvent du nord au sud en passant par Xi' an, Shanghai, Suzhou, Guilin, Canton et repartent de Hong Kong, quelquefois en sens inverse. On voit bien que le deuxième itinéraire est pratiquement une version raccourcie du premier, sans l'extension à Canton et Hong Kong, donc la durée du séjour passe de 15 jours à 13 jours en général. Par contre, quelques nouvelles étapes sont ajoutées telles que Pingyao et Tongli, villages traditionnels, en contraste avec les grandes métropoles chinoises. De plus, Shanghai est positionné après Guilin et Yangshuo pour devenir la dernière étape de l'itinéraire grâce au vol direct Shanghai-Paris.
«Aux confins du Yunan» (La Maison de la Chine), "Sur la route du thé, des chevaux et du Tibet» (Voyageurs du Monde) et "Yunnan, sur la route du thé » (Asia) programment la région sud-ouest de la Chine, où vivent de nombreuses minorités avec leurs habits traditionnels et leurs modes de vie folkloriques en correspondance avec le paysage montagneux ainsi que la culture et le marché du thé. Avec l'ouverture de la ligne aérienne directe en 2014, Kunming, chef-lieu de la province du Yunnan, sert du lieu d'entrée et de départ pour cet itinéraire, ce qui implique des lieux avec des caractéristiques typiquement ethniques au sein de la province (voir Carte 2, l'Itinéraire 3). La durée du voyage varie entre 13 à 17 jours selon le programme. « Caravanes de la Soie » (La Maison de la Chine) et «La Route de la Soie en Chine» (Voyageurs du Monde) empruntent l'ancienne Route de la Soie que traversaient des caravanes chargées de soie, d'épices, de fourrures et de parfums pour des échanges économiques et culturels entre l'Orient et l'Occident. Aujourd'hui, les touristes français revivent ce long cheminement qui présente des paysages désertiques avec le mode de vie typique des minorités Hui et Uighur dans les confins nord-ouest de l'empire. L'itinéraire commence par Xi'an, ancienne capitale chinoise desservie par des vols directs avec Paris depuis 2014, puis parcourt la région autonome du Ningxia Hui et celle du Xinjiang Uighur, pour repartir de Xi'an ou de Chengdu pour la France, le voyage dure entre 12 et 17 jours. À part ces deux itinéraires assez suivis par les touristes français, la province du Sichuan et la région tibétaine sont aussi entrées dans les catalogues touristiques des Français, mais leur proportion reste relativement faible par rapport aux itinéraires présentés avant.

\section{B. Évolution des itinéraires des touristes français en Chine}

Des entretiens réalisés avec CYTS (China Youth Travel Service) de Canton, GZL (Guangzhou Travel Service) et CITS (China International Travel Service) de Guilin permettent de tracer l'évolution des itinéraires des touristes français en Chine, ce qui, avec la statistique du nombre de visiteurs français en Chine (Figure 1), correspondent au développement du marché touristique dans ce pays. Au cours des 40 dernières années, le tourisme chinois a vécu une transition du « tourisme planifié » au « tourisme de marché », en passant par quatre phases avec des 


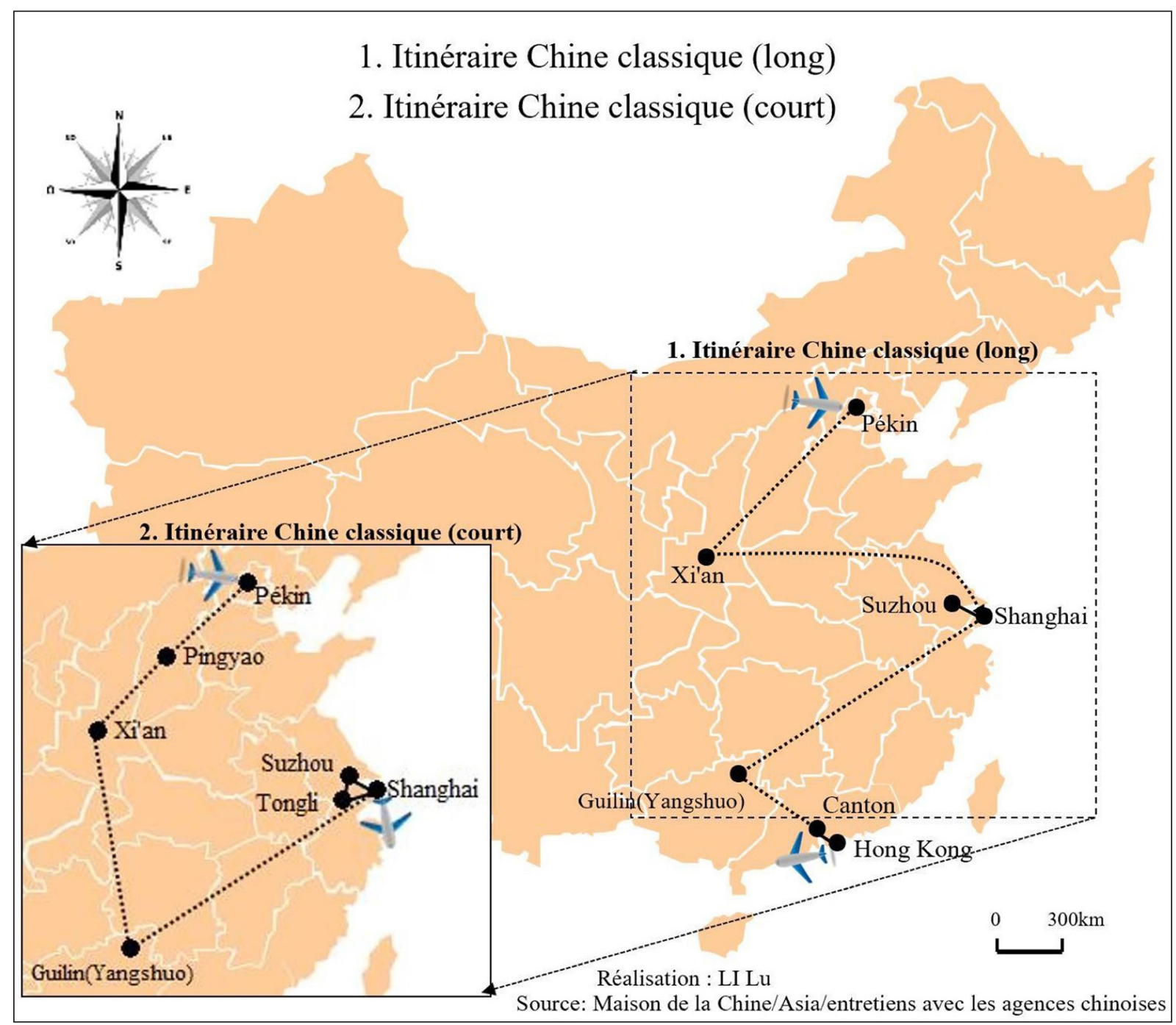

Carte 1. Itinéraires Chine classique

caractéristiques spécifiques, qui nous aident à mieux comprendre l'évolution du tourisme récepteur ainsi que le tourisme des Français en Chine.

\section{Au début de la réforme et de l'ouverture (1978- 1991)}

Le tourisme récepteur de cette époque n'a pas complètement abandonné son habit politique et sa fonction diplomatique pour l'État chinois, qui nécessita de rétablir sa réputation internationale à travers l'accueil des « invités étrangers » dans des lieux et des sites spécialement choisis et aménagés au sein du pays (Song, 2006). Pourtant, sa fonction économique fut reconnue surtout en tant que source importante de recettes en devises. Sous le monopole de l'État, le tourisme commença à revêtir une importance dans l'économie nationale : d'une part, attachées au gouvernement chinois, les trois agences de voyages, le CTS, le CITS et le
CYTS collaboraient et se partageaient l'ensemble du marché touristique, en particulier la partie du tourisme récepteur; d'autre part, la création de 1'Administration Nationale du Tourisme de Chine en 1982 et de ses nombreuses branches provinciales et municipales, ainsi que l'élaboration et l'application d'un grand nombre de politiques et de règlements à l'égard du tourisme, reflètent l'accent mis sur le secteur du tourisme et le contrôle strict de l'État chinois. L'espace ouvert aux touristes étrangers se limita d'abord à quelques grandes villes telles que Pékin, Shanghai, Canton, Xi'an, centres historiques, politiques et économiques avec des infrastructures adaptées à l'accueil des « invités étrangers » et des sites touristiques identifiés à l'image véhiculée de la Chine. Des villes touristiques connues pour leur beau paysage qui représente la Chine idéale et poétique sont aussi entrées dans les itinéraires : nous pouvons citer Guilin par exemple, qui est parmi les premiers 


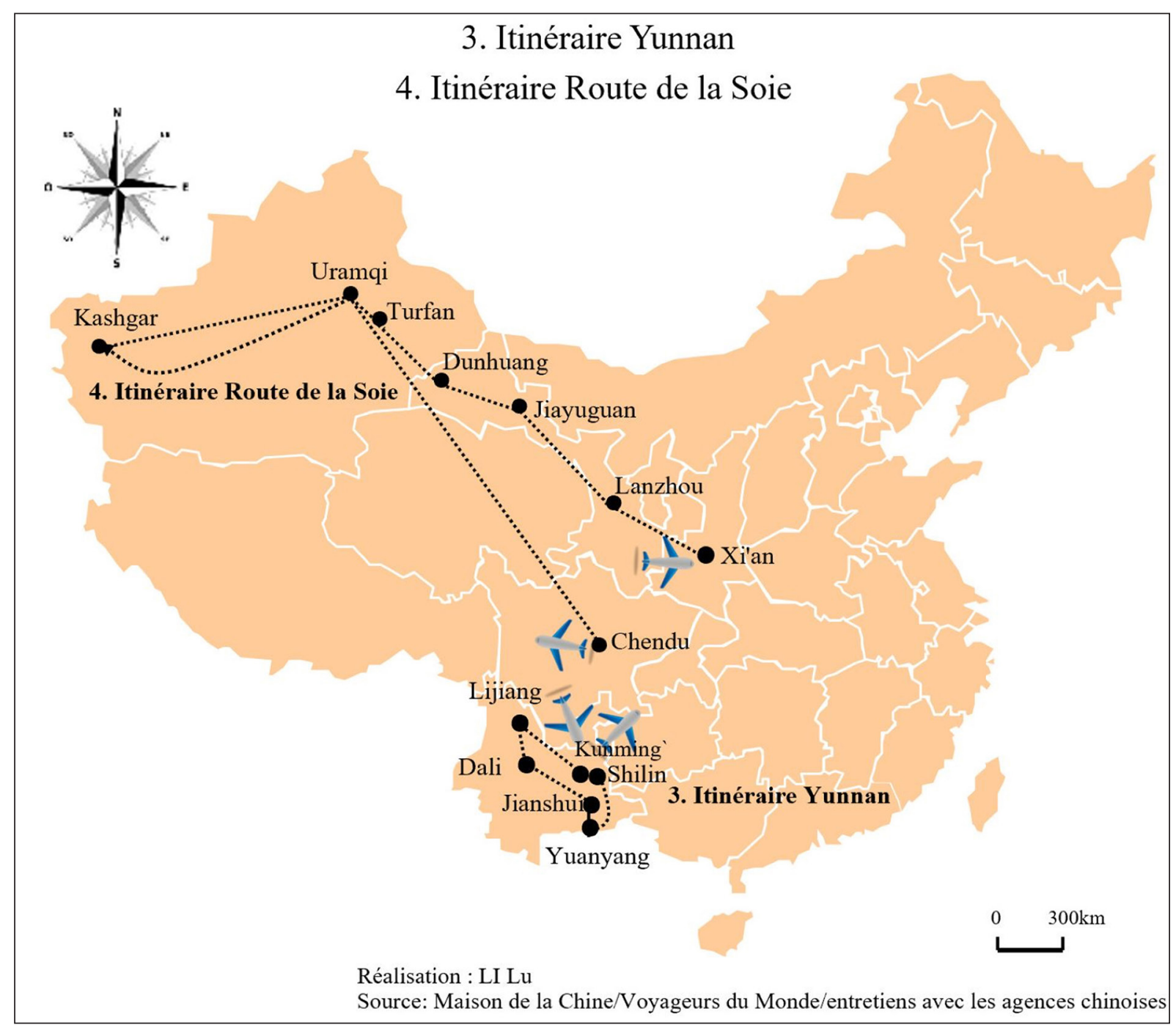

Carte 2. Itinéraires Chine intérieure

lieux à être rouverts aux touristes étrangers en 1973 (Urry, 1990). Ainsi, contenant les sites les plus typiques, des circuits classiques tels que l'Itinéraire Chine classique (long) présenté sur la Carte 1 ont vu le jour et ce circuit constitue depuis sa création le produit vedette pour les touristes français en premier contact avec la Chine, avec quelques modifications au niveau du choix des étapes et de la durée du séjour au cours de ces années. Au début, le circuit commençait souvent à Hong Kong, grâce à son accessibilité par des vols internationaux avec la France, pour remonter du sud au nord par Canton, Guilin, Shanghai, Xi' an et enfin Pékin pour repartir pour Paris, puisque la capitale chinoise a ouvert le vol direct avec la France depuis 1973.

\section{L'essor du tourisme récepteur (1992-2002)}

En 1992, le Conseil des Affaires d'État de Chine a promulgué « la Décision de Promouvoir le Secteur
Tertiaire », une politique qui précise davantage l'importance du tourisme dans le secteur tertiaire et détermine une attention particulière sur le développement du tourisme. Le marché s'est substitué petit à petit à la place de l'État pour décider des systèmes de tarification, les entreprises touristiques ont ainsi acquis plus d'autonomie. L'État ne fut plus propriétaire du secteur du tourisme, mais plutôt l'administrateur dirigeant à travers l'établissement des lois et des règlements ainsi que l'élaboration des mesures et des projets de planification pour promouvoir le développement du tourisme.

Pendant cette période, le tourisme récepteur a connu un essor rapide avec des arrivées internationales et des retombées économiques croissantes. Le nombre d'arrivées annuelles de visiteurs français est passé de 116000 à 222000 en 10 ans. $\mathrm{Xu}(2004)$ a listé dans son étude les premières dix destinations chinoises pour les touristes fran- 


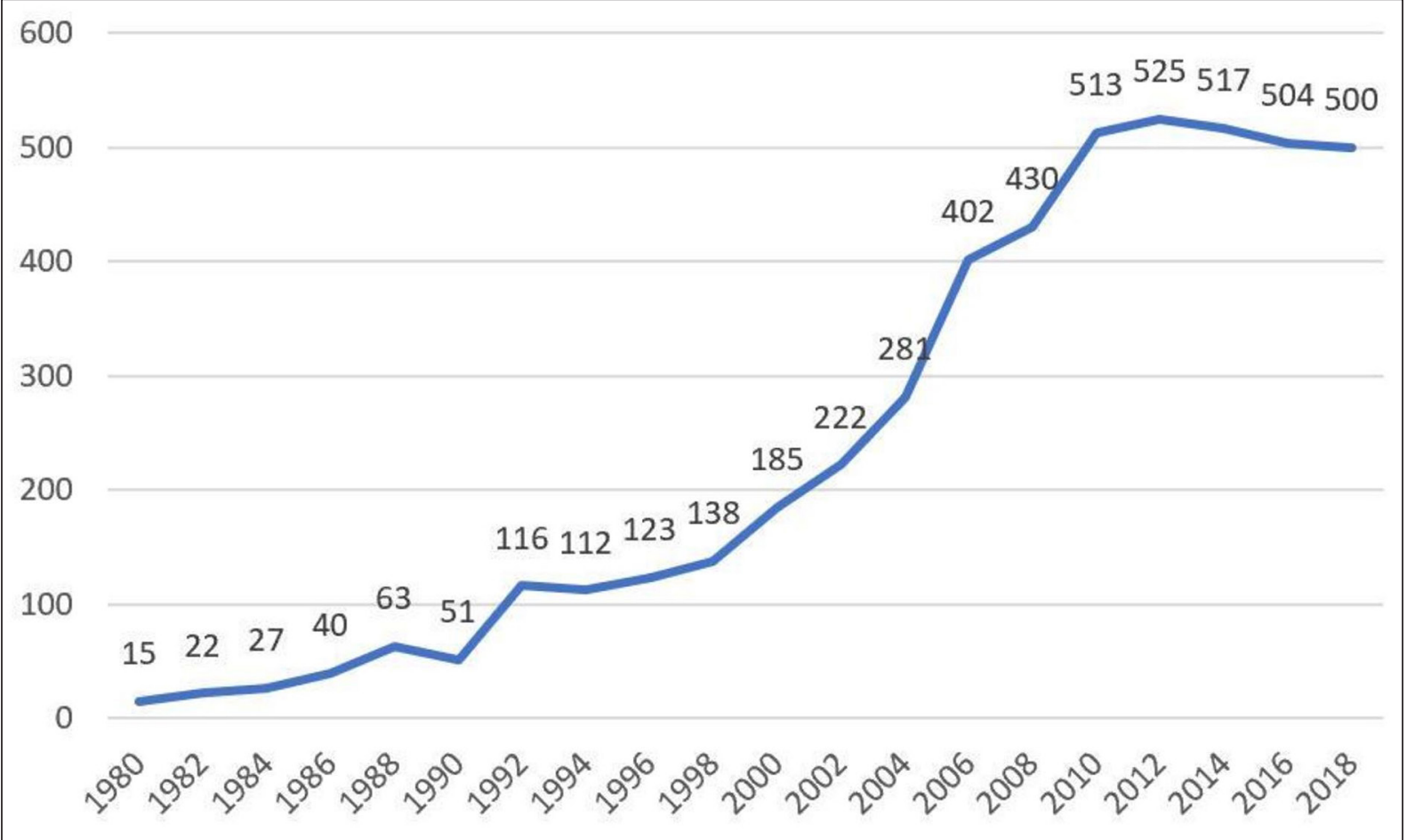

Figure 1. Nombre d'arrivées de visiteurs français en Chine entre 1980-2018 (unité : 1000 arrivées) - Source : Ministère de la Culture et du Tourisme de la Chine

çais : les trois grandes portes aériennes Pékin, Shanghai et Canton se classaient parmi les trois destinations les plus fréquentées sur l'ensemble du territoire chinois. Le Shaanxi (dont le cheflieu est Xi'an), le Guangxi (dont le chef-lieu est Guilin), le Jiangsu (avec Suzhou et Nankin comme atouts touristiques), le Zhejiang (dont quelques villages pittoresques autour de Shanghai) et le Yunnan (connu par le paysage folklorique des minorités) étaient toujours dans la liste avec leurs places plutôt stables. Néanmoins, l'apparition de nouvelles destinations dans ce palmarès comme le Hubei et Chongqing en 1997 s'explique par les travaux liés à la construction du célèbre Barrage des Trois Gorges. Cette construction allait causer la submersion de la section la plus belle du fleuve Yangzi, section que tout le monde voulait visiter avant la fin des travaux. Ainsi, à part les itinéraires classiques qui étaient toujours les plus choisis par les touristes français, quelques nouveaux produits tels que la croisière sur le fleuve Yangzi et le Yunnan sont entrés dans le catalogue touristique. En plus, des extensions se sont ajoutées à l'itinéraire classique comme la visite aux jardins des anciens lettrés chinois à Suzhou et celle dans le village d'eau de Zhouzhuang, en vue de varier les produits touristiques et de renforcer les atouts de la destination chinoise.

\section{L'épanouissement du tourisme chinois (2002-} 2010)

Depuis que la Chine est affiliée à l'Organisation Mondiale du Commerce en 2002, l'ouverture du marché du tourisme a atteint un niveau plus élevé. Le tourisme intérieur et le tourisme émetteur ont connu une augmentation considérable, vu que le niveau de vie des Chinois s'est beaucoup amélioré et que de plus en plus de destinations sont ouvertes au public chinois. Bien que le tourisme récepteur ne fût plus la préoccupation dans le développement du secteur touristique avec la réorientation de l'économie nationale vers l'encouragement de la demande intérieure, le nombre d'arrivées de touristes internationaux y compris des Français était toujours en augmentation étant donné les relations multipliées entre les pays et l'amélioration des prestations touristiques et de la qualité d'accueil. Le nombre d'arrivées annuelles de visiteurs français a atteint 513000 en 2010. Depuis 2002, les TO étrangers ont le droit d'ouvrir leurs propres bureaux en Chine, leur permettant de contrôler l'ensemble des chaînes des prestations touristiques, y compris l'accueil des touristes sur la destination, qui était réservé auparavant aux agences réceptives chinoises. Donc, il s'agit d'une période de maturation du marché touristique et d'amélioration des produits touristiques pour les touristes étrangers. 
Les itinéraires classiques persistaient toujours pendant ce temps, avec des étapes et des sites ajoutés ou supprimés ou encore remplacés. Par exemple, entre Pékin et Xi'an, on a ajouté une nouvelle étape, Pingyao dans la province du Shanxi. Inscrite au patrimoine mondial de l'UNESCO en 1997, cette ville historique très bien conservée où l'architecture traditionnelle chinoise domine était connue petit à petit par les touristes étrangers. Il en était de même pour Luoyang, situé dans la province du Henan et centre du bouddhisme avec des temples et des grottes, qui devenait aussi un lieu emblématique. Par contre, Zhouzhuang, village d'eau connu pour être la plus belle ville de canaux de Chine depuis plus d'une décennie, est innondé par de nombreux touristes chinois qui rendent l'expérience moins agréable pour les autres. Ainsi, Tongli, antique bourg d'eau, à équidistance de Zhouzhuang et de Suzhou, offrant des coins tranquilles loin des foules pour admirer les canaux et l'architecture des maisons aux murs blanchis à la chaux, a remplacé petit à petit Zhouzhuang dans l'itinéraire classique des TO. Les touristes français commençaient leur visite plutôt à Pékin pour descendre du nord au sud, et ils avaient tendance de raccourcir leur durée de voyage en supprimant la partie sud (Canton et Hong Kong) pour repartir de Shanghai pour la France. De plus, les produits touristiques pour les touristes français se diversifiaient avec l'apparition des nouveaux itinéraires tels que la route de la soie et la région tibétaine, etc. Avec la maturation du secteur touristique en Chine, le nombre de touristes étrangers visitant la Chine par eux-mêmes au lieu de passer par les agences de voyages augmenta.

\section{Un marché diversifié et personnalisé (depuis 2011)}

Depuis le déclenchement du $12^{\mathrm{e}}$ Plan quinquennal de la Chine (2011-2015), le secteur du tourisme est entré dans une nouvelle phase en revêtant des objectifs économiques et sociaux. Il joue un rôle plus actif dans le service de la vie humaine, la promotion de la culture, l'éducation, etc. (Tang, 2014). La venue des TO étrangers sur le marché du tourisme a accentué la concurrence et a entraîné la perte de parts de marché d'anciennes agences de voyages d'état au niveau du tourisme récepteur en Chine, ce qui a également entrainé une réorientation des activités de ces dernières. Ainsi, même si le nombre de visiteurs français reste plutôt stable avec environ 500000 arrivées annuelles, les TO français, surtout ceux qui ont créé leur propre bureau en Chine, ont acquis une plus grande part du marché.

Le marché touristique sur la Chine devient de plus en plus diversifié et complexe, ce qui se traduit par des offres sous maintes formes et couvrant un plus grand territoire ainsi qu'une augmentation du degré de la personnalisation dans ces offres. Les itinéraires classiques sont toujours en service, avec des variations raccourcies et simplifiées. On apprécie moins un programme compact visant à parcourir autant de sites que possible en un temps limité, la visite se déroule à un rythme plus modéré et agréable. Ainsi, l'itinéraire Chine classique (court) de la carte 1 occupe une part plus importante qu'avant. Des prestations « séjour » dans une métropole telle que Pékin, Shanghai et Hong Kong, qui permettent de découvrir les essentiels à son propre rythme et d'éviter la fatigue du voyage entre les étapes, sont nombreuses dans les catalogues. Des produits « à la carte » ou encore « sur mesure » sont plus présents avec des services personnalisés. On tend à partir en petits groupes et visiter la destination plus en profondeur. Les TO fournissent aux touristes des prestations flexibles, avec des assemblages en petits packages. Par exemple, si un touriste séjournant à Shanghai veut découvrir un peu les alentours, il peut choisir une excursion à Suzhou pour visiter ses fameux jardins, ou encore faire un petit voyage dans la province voisine du Anhui pour découvrir la Montagne Jaune ; une extension à Hong Kong peut aussi être ajoutée au circuit si le touriste veut visiter d'autres lieux plutôt que de rester deux jours à Canton. Des itinéraires incluant des lieux auparavant considérés comme « hors des sentiers battus », tels que la Route de la Soie, le Yunnan et le Tibet, attirent plus de touristes français qu'avant, les touristes d'aujourd'hui s'intéressent de plus en plus à la découverte de la multidimension de la culture chinoise à travers l'exploration approfondie dans la Chine « intérieure ». Des prestations avec un thème spécifique, qui implique la participation des touristes sont de plus en plus demandées alors que celles qui ne s'adaptent plus à l'actualité sont retirées du marché, la croisière sur le Fleuve Yangzi est un exemple.

L'évolution des itinéraires touristiques pour les touristes français en Chine pendant ces dernières 40 années nous fournit un microcosme du développement du tourisme récepteur dans ce pays. Au cours de la transition des fonctions politiques du tourisme 
vers les fonctions économiques et sociales, la prépondérance du gouvernement s'est progressivement affaiblie et a été remplacée par le marché. Le choix et l'ouverture des lieux touristiques sont également passés progressivement d'une décision de l'état en fonction de ses besoins de propagande à une offre issue de l'influence commune du gouvernement et du marché, et aujourd'hui, plutôt déterminée par le comportement d'un marché adapté à « une clientèle plus diversifiée, personnalisée, sélective et participative » $(\mathrm{Xu}, 2003)$. De plus, la diversification des produits touristiques ne se limite pas au contenu de la visite, l'organisation varie aussi. La part du circuit accompagné en groupe est en train d'être grignotée par des prestations avec plus d'autonomie pour les touristes.

\section{ANALYSE DE L'ENQUÊTE AUPRÈS DES TOURISTES FRANÇAIS EN CHINE}

Après l'analyse des offres touristiques, nous avons mené une enquête ${ }^{3}$ auprès des touristes français en Chine en vue de vérifier ce qu'ils font réellement pendant leur voyage.

\section{A. Les profils des touristes français et les caractéristiques de leurs séjours}

Après le traitement des données des 515 questionnaires remplis par les touristes français de passage à Yangshuo, nous pouvons essayer de dessiner leurs profils : les Français de 45 à 64 ans constituent les principaux visiteurs : ils constituent la moitié de notre échantillon, 56,6\% des touristes enquêtés sont mariés ou vivent en couple, et ils voyagent souvent en couple ou en famille. $71 \%$ ont fait des études supérieures, $45,4 \%$ travaillent dans la catégorie " professions libérales, cadre d'entreprise, de la fonction publique, profession intellectuelle supérieure », et $36 \%$ ont déclaré un revenu mensuel brut de plus de 3000 euros. Ainsi, cette clientèle concerne plutôt la classe moyenne française. Deux touristes sur cinq proviennent de la région parisienne. Leurs séjours en Chine varient de 5 jours à 62 jours au maximum, avec plus de la moitié voyageant pendant 12-15 jours. En général, un groupe touristique organisé comprend 14 personnes; ils logent le plus souvent dans des hôtels de deux ou trois étoiles. L'avion est le moyen de transport le plus utilisé $(96,1 \%)$ pour leur voyage en Chine. Cela peut s'expliquer par le fait que la Chine est un vaste pays et que l'avion peut paraître un choix raisonnable pour parcourir les distances importantes entre les différentes étapes. Le train $(80,2 \%)$ et l'autocar $(77,9 \%)$ se classent respectivement en deuxième et troisième rangs parmi les moyens de transport les plus choisis pour certaines étapes. Le chemin de fer est développé en Chine, c'est un atout incontestable pour les Français qui ont le goût et l'habitude pour ce moyen de transport. Le car touristique est généralement adopté par les groupes touristiques durant leur séjour en Chine, et les touristes voyageant en groupe organisé sont majoritaires dans notre échantillon.

\section{B. Les lieux touristiques fréquentés par les touristes français en Chine}

À partir de la réponse des enquêtés, 69 lieux touristiques ont été retenus. Nous nous apercevons (Tableau 1) que les lieux les plus fréquentés par les touristes français enquêtés sont toujours des lieux touristiques classiques, ce qui confirme notre analyse des produits touristiques et des entretiens avec les professionnels. Nous pouvons citer par exemple Yangshuo, Guilin ${ }^{4}$, Pékin, Xi' an, Shanghai, Hongkong, Canton, Suzhou comme les lieux les plus fréquentés avec plus de 200 touristes enquêtés qui les ont inclus dans l'itinéraire de leur voyage en Chine. Les sept premiers lieux accueillent plus de la moitié des déplacements de notre échantillon. On voit une rupture radicale entre le $9^{\mathrm{e}}$ et le $10^{\mathrm{e}}$ rang dans la liste : Pingyao dénombre 195 arrivées alors que Tongli en compte seulement 83. Ensuite, les trois villes du Yunnan occupent respectivement les $12^{\mathrm{e}}, 13^{\mathrm{e}}$ et $15^{\mathrm{e}}$ place, avec Hangzhou au $14^{\mathrm{e}}$ rang.

Nous remarquons que les principaux lieux fréquentés sont plutôt situés dans la partie est de la diagonale symbolique divisant la Chine du nord-est au sud-ouest (Carte 3). Nommée « Ligne Heihe-Tengchong » ou "Ligne $\mathrm{Hu}$ » en mémoire de son créateur Monsieur HU Huanyong, cette diagonale reliant Heihe dans la province du Heilongjiang et Tengchong dans la province du Yunnan, divise la Chine en deux parties selon la densité de la population chinoise : $94 \%$ de la population chinoise vit dans le sud-est qui ne couvre que $43,8 \%$ de la superficie chinoise. Elle représente également la distinction au niveau du développement économique et de l'urbanisation du pays. Le tourisme respecterait cette ligne avec les lieux les plus fréquentés concentrés dans la partie est. Notons que le choix du lieu d'enquête qui se situe dans la 


\begin{tabular}{|c|c|c|c|c|c|}
\hline Rang & Lieux & Nombre d'arrivées & Rang & Lieux & Nombre d'arrivées \\
\hline 1 & Yangshuo & 514 & 16 & Macao & 44 \\
\hline 2 & Guilin & 482 & 17 & Chengde & 41 \\
\hline 3 & Pékin & 466 & 18 & Luoyang & 40 \\
\hline 4 & Xi'an & 440 & 19 & Xiamen & 26 \\
\hline 5 & Shanghai & 420 & 20 & Chengdu & 23 \\
\hline 6 & Hong Kong & 326 & 21 & Chongqing & 20 \\
\hline 7 & Canton & 262 & 22 & Guiyang & 15 \\
\hline 8 & Suzhou & 249 & 23 & Lanzhou & 15 \\
\hline 9 & Pingyao & 195 & 24 & Huangshan & 14 \\
\hline 10 & Tongli & 83 & 25 & Shenyang & 9 \\
\hline 11 & Datong & 79 & 26 & Sanya & 7 \\
\hline 12 & Lijiang & 66 & 27 & Shenzhen & 7 \\
\hline 13 & Kunming & 56 & 28 & Ping'an & 7 \\
\hline 14 & Hangzhou & 49 & 29 & Zhouzhuang & 6 \\
\hline 15 & Dali & 45 & 30 & Lassa & 6 \\
\hline
\end{tabular}

Tableau 1. Les 30 premiers lieux touristiques chinois les plus fréquentés par les Français présents à Yangshuo

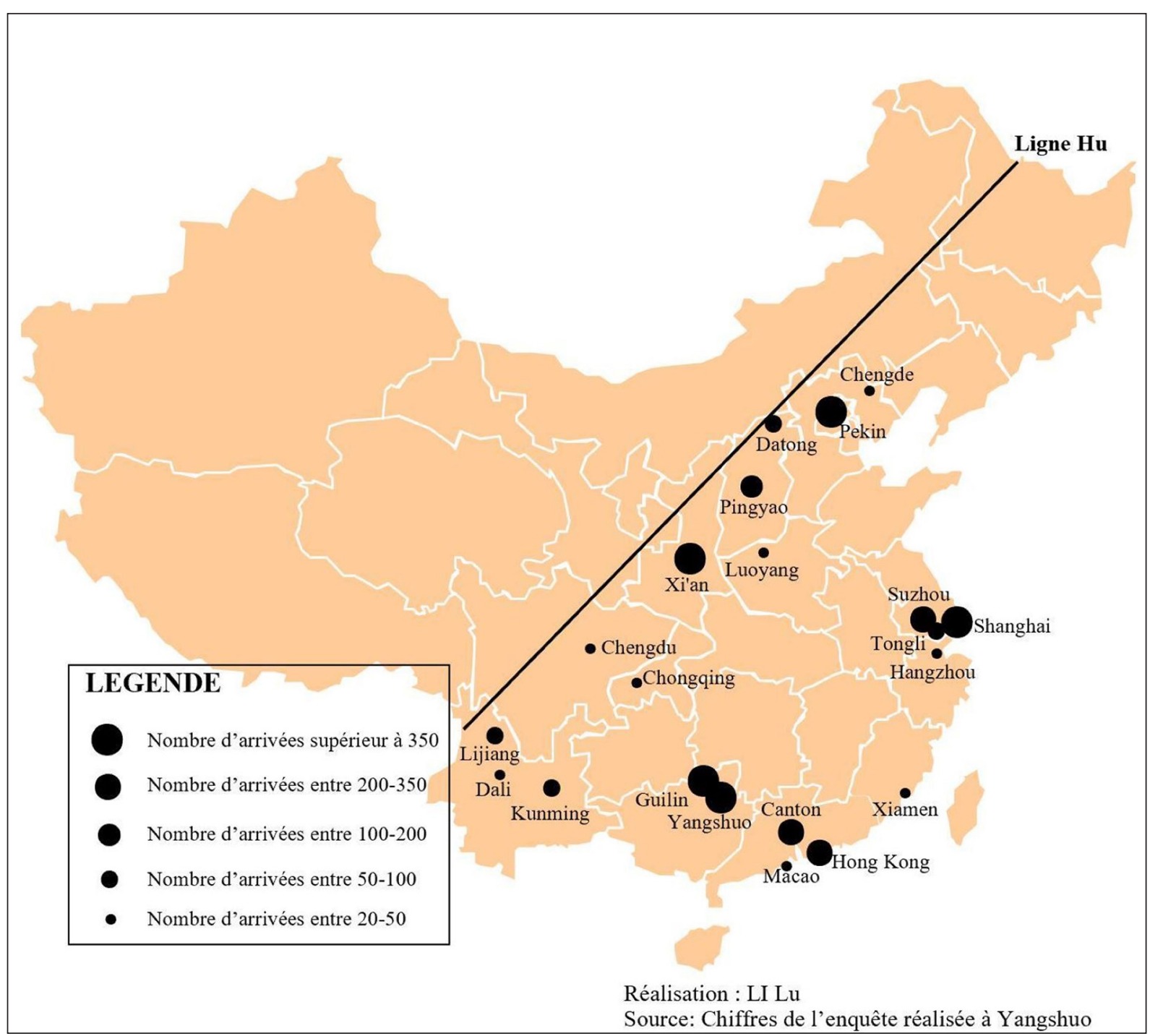

Carte 3. Répartition des lieux touristiques selon les arrivées des 515 touristes français en Chine 
partie sud peut induire un biais d'échantillonnage, puisque Yangshuo et Guilin ne font pas partie des itinéraires du nord-ouest selon les circuits présentés dans la section précédente. Cependant, nous avons interrogé dans notre échantillon 90 touristes en voyage auto-organisé, parmi lesquels certains sont venus plusieurs fois en Chine, et qui ont très rarement mis le pied dans la partie nord-ouest. Dès lors l'enquête confirme que la partie ouest chinoise n'est pas encore une destination très fréquentée par des touristes français, au moins pour ceux qui ne passent pas par les agences de voyages.

Selon nos statistiques, parmi les dix premiers lieux touristiques les plus fréquentés par les touristes français, sept se classent parmi des grandes villes comprenant les trois plus grandes métropoles chinoises : Pékin, Shanghai et Canton. Toutes ces villes occupent une place importante dans l'économie, la politique ainsi que l'histoire et la culture de la Chine. Les trois autres lieux dans ce palmarès sont des districts (Yangshuo, Pingyao et Tongli), qui possèdent toujours une population importante et un dynamisme économique, surtout au niveau de l'accueil des touristes domestiques et internationaux puisqu'ils sont des hauts lieux touristiques. À travers ces résultats, nous constatons que les villes occupent une place dominante parmi tous les lieux touristiques. Nous comptons dans la liste 39 villes chinoises qui représentent $78,5 \%$ des arrivées touristiques, soit environ trois quarts de la fréquentation totale de nos enquêtés. On trouve encore des districts, sous l'administration des villes, qui prennent le tourisme comme pilier économique : nous pouvons citer Yangshuo, Pingyao comme exemples ; certains sont situés en périphérie des villes et profitent ainsi des arrivées touristiques importantes : Tongli (tout près de Suzhou) et Zhouzhuang (à côté de Shanghai) en sont des exemples. Par conséquent, la fréquentation des villes est encore très présente pour les touristes français en Chine. Cela pourrait s'expliquer d'abord par la commodité de la ville au niveau du transport, du logement et de la restauration. Ensuite, il s'agit d'un choix historique du tourisme chinois : les premiers lieux choisis pour l'accueil des touristes étrangers sont des villes relativement développées dont l'infrastructure est mieux adaptée pour l'aménagement touristique, et ces choix sont renforcés davantage par l'investissement accentué et continu sur ces villes au cours du développement du tourisme. Néanmoins, il ne faut pas négliger les attraits de ces villes mêmes : les habitants, leurs modes de vie et leurs traditions constituent une partie importante de ce que les touristes français souhaitent voir pendant leur visite en Chine.

Parmi le reste des 30 lieux touristiques, certains se répartissent sur un territoire immense hors des villes et possèdent soit un paysage naturel spectaculaire (ex : Taishan, Wutaishan, Emeishan, Shaolin, Yuanyang, Longji...), soit des traditions folkloriques typiques (ex : Ping'an, Zhaoxing, Xijiang, Chejiang, Kaili, Dazhai, Basha, Paikai...), ou bien les deux ensemble (ex : Xishuangbanna, Jiuzhaigou...). Cela implique que les hauts lieux qui représentent une culture typique de la Chine attirent souvent les touristes français; mais le nombre d'arrivées n'est pas si important que pour les villes chinoises.

Ces résultats confirment les conclusions tirées par l'analyse des catalogues touristiques et celle des entretiens avec les professionnels : jusqu'à présent, les villes chinoises emblématiques sont toujours « le plat principal » pour les touristes français, qui se concentrent surtout dans la partie est de la Chine, tandis que des lieux « reculés », situés dans l'ouest de la Chine présentant les cultures des ethnies minoritaires, sont servis plutôt comme «le dessert ».

\section{Les activités touristiques des touristes fran- çais en Chine}

Nous avons divisé les activités touristiques en 12 catégories à partir des activités possibles à faire pendant le séjour en Chine, et le résultat est présenté dans la Figure 2.

Selon nos statistiques, il est évident que les activités touristiques les plus pratiquées résident dans la découverte : " admirer des sites naturels », « visiter des sites et monuments culturels et historiques (musées, temples, palais ou tombeaux royaux...) ", " découvrir la gastronomie chinoise », « voir des spectacles ou des manifestations culturelles et folkloriques », " découvrir la montagne », et « apprendre le chinois et les arts chinois (calligraphie, arts martiaux, Taiji...) ». Les deux premières modalités, c'est-à-dire, « admirer des sites naturels » et « visiter des sites et monuments culturels et historiques (musées, temples, palais ou tombeaux royaux...) » concernent pratiquement tous les touristes français venant en 


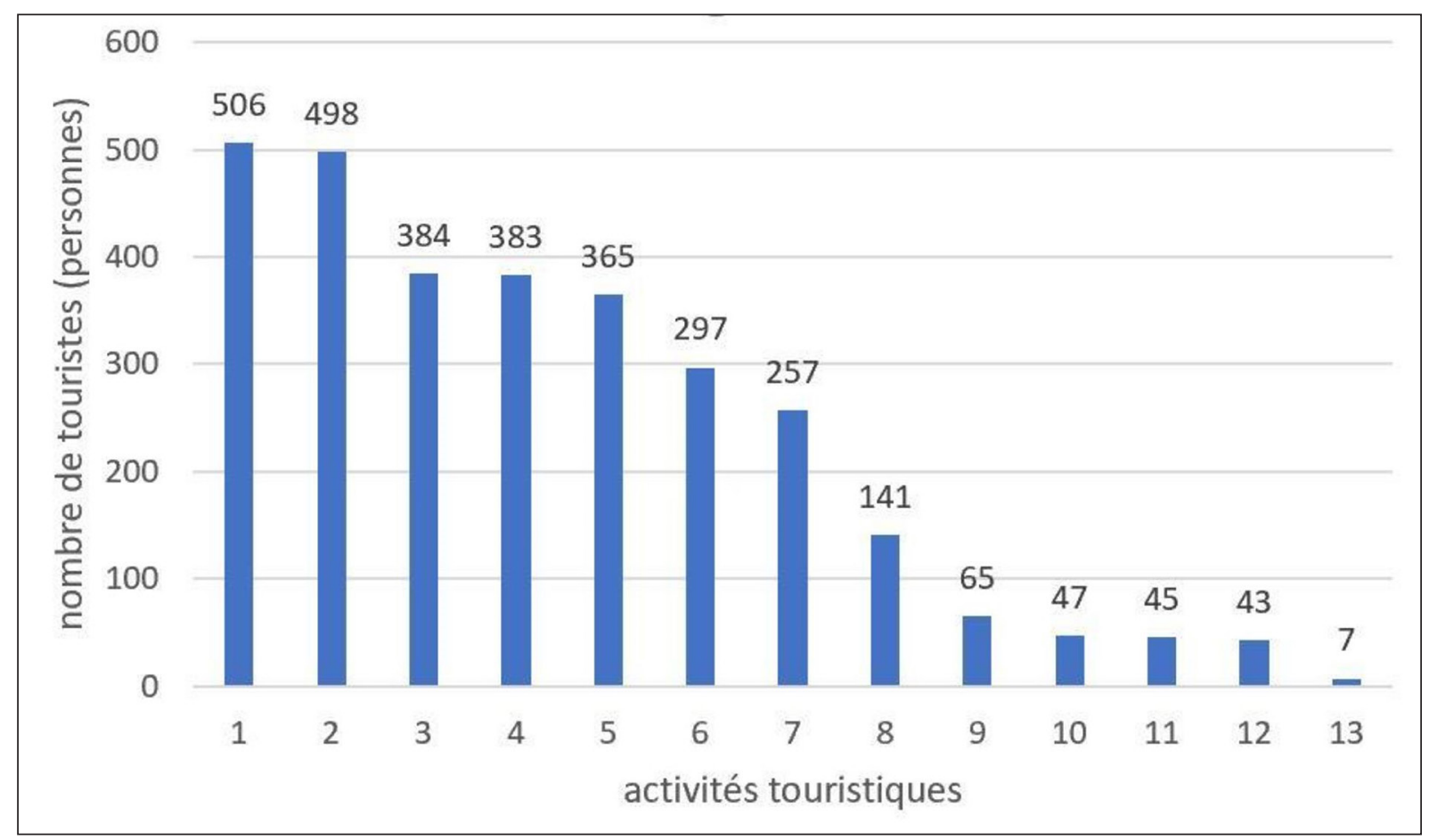

Figure 2. Répartition des touristes français selon les activités touristiques pratiquées en Chine. Activités touristiques : 1. Admirer le paysage (sites naturels) ; 2. Visiter des sites et monuments culturels et historiques ; 3. Se promener en ville; 4. Découvrir la gastronomie chinoise ; 5. Voir des spectacles ou des manifestations culturelles et folkloriques ; 6. Faire du shopping ; 7. Aller à la rencontre des habitants dans les quartiers et la vie courante ; 8. Découvrir la montagne ; 9. Visiter parents ou amis ; 10. Apprendre le chinois et les arts chinois ; 11. Exercer des activités sportives; 12 . Aller sur la plage ou se baigner ; 13. Autres

Chine ; les $4^{\mathrm{e}}$ et le $5^{\mathrm{e}}$ modalités, soit « découvrir la gastronomie chinoise » et " voir des spectacles ou des manifestations culturelles et folkloriques ", sont présentes chez les trois quarts des enquêtés ; plus d'un quart des enquêtés ont découvert la montagne. Il est important de souligner qu'en Chine les montagnes ne sont pas destinées au ski ou à d'autres sports d'hiver, mais plutôt pour faire des visites de découverte et en même temps des exercices physiques. Dans la culture chinoise, être très proche du ciel signifie qu'on s'approche de plus en plus de l'éternité. Donc, les hautes montagnes servent aux rites sacrés et l'ascension s'apparente à une sorte de pèlerinage, durant lequel on subit une épreuve physique et spirituelle. Ainsi, l'activité de montagne peut entrer à la fois dans la découverte et le sport. Par contre, le nombre des pratiquants des activités restantes ne dépasse pas la moitié des touristes enquêtés excepté « la promenade en ville» et « le shopping », pratiques du tourisme de ville aussi très présent dans cette destination. Le repos et le sport («aller sur la plage ou se baigner », « exercer des activités sportives ») ainsi que la visite à des proches (« visiter parents ou amis ») ne touchent qu'un dixième de notre échantillon. Ce résultat confirme la dominance de la découverte dans les pratiques des touristes français en Chine au bout de 40 ans après l'ouverture de cette destination. Contrairement à leurs pratiques en France métropolitaine, la mer en Chine n'est pas aussi attrayante pour les touristes français : seuls $9,0 \%$ de notre échantillon étaient allés sur la plage. «L'apprentissage du chinois et des arts chinois » $(9,1 \%)$ ainsi que des "activités sportives" $(8,7 \%)$ sont aussi relativement peu pratiqués par les touristes français en Chine.

À partir de ces résultats, nous pouvons remarquer que la découverte des touristes français en Chine concerne principalement le paysage, les monuments historiques, la cuisine, les spectacles folkloriques, la langue et les arts chinois... tout cela relève de l'altérité chinoise, l'originalité de la Chine qui distingue ce pays des autres destinations touristiques. Le tourisme consiste à changer de place et à affronter l'altérité (Violier, 2000). L'altérité n'existe qu'ailleurs, on choisit cet « ailleurs » parce qu'il possède la qualité différente de celle du lieu de vie et en même temps répond à son intentionnalité. Ainsi, les activités des touristes français en Chine 
sont peu liées à la mer ou au sport, parce qu'ils ont beaucoup de choix, même de meilleure qualité, en France ou dans les pays voisins. La Chine n'est pas destinée à ce genre d'activités. Ils choisissent la Chine pour la grande qualité chinoise, autrement dit l'altérité chinoise, unique dans le monde qui ne se trouve pas ailleurs. François Jullien (1991) l'a définie comme une autre civilisation qui n'a pas connu de rupture ni d'importante mutation, qui est restée fidèle à ses origines et à ses valeurs, que "nous nous plaisons à imaginer de si loin comme les plus subtils raffinements d'une autre culture ». De ce fait, les touristes français sont fascinés pour la campagne et la vie des paysans à Yangshuo, les spectaculaires armées en terre cuite à Xi'an, le grandiose palais impérial de Pékin, les exquis jardins des anciens lettrés à Suzhou, etc. Mais voir de grandes usines ou des grandes autoroutes, cela ne les intéresse pas vraiment, puisqu'ils ont les mêmes chez eux. De plus ils sont parfois confrontés à un " choc » en voyant les gratte-ciels et des centres villes éclairés rappelant fortement les États-Unis. La raison fondamentale de ces réactions réside dans le fait que cela n'est pas ce qu'ils attendent de la Chine. Il ne s'agit pas forcément de la réalité chinoise telle qu'elle est construite dans l'imaginaire populaire (Villard, 2006).

\section{CONCLUSION}

Notre recherche vise à comprendre les pratiques des touristes français en Chine. L'analyse des catalogues touristiques et des entretiens avec des professionnels ont permis d'illustrer le marché des touristes français ainsi que son évolution depuis l'ouverture de cette destination. Une enquête auprès de ces touristes a confirmé les choix des lieux fréquentés et a dévoilé leurs activités principales durant leur visite. Les résultats montrent que les touristes français fréquentent principalement des hauts lieux touristiques classiques, souvent des villes avec une importance économique, politique et culturelle, situées dans l'est de la Chine. En correspondance avec les lieux fréquentés, leurs activités sont étroitement liées à la découverte, notamment en ce qui concerne l'altérité chinoise, qui se traduit par sa civilisation plusieurs fois millénaire incarnée dans le paysage particulier de ce territoire et chez les peuples chinois avec leur vie très différente. $\mathrm{Si}$ au début le tourisme des Français en Chine a été un choix politique de l'État chinois, il est également le résultat d'un marché au fil du développement du tourisme : 1'attrait représentatif de ces lieux mêmes et les goûts des touristes français jouent un rôle dans le processus du choix pour les prestations touristiques des TO. Ainsi, le développement du tourisme en Chine apporte des modifications sur les choix des lieux comme l'intégration de Pingyao, Tongli dans des circuits classiques et l'apparition des nouveaux itinéraires à l'ouest Chine, mais également le repli de certains lieux comme Zhouzhuang, ce qui représente le résultat des choix du marché dans le processus du développement du tourisme chinois. Avec la mondialisation et le développement de l'informatique, la Chine ne serait plus un pays inconnu pour les nouvelles générations françaises. L'altérité chinoise, issue du mystère inconnu, sera-t-elle à redéfinir dans le nouveau contexte ? Est-elle aussi attrayante pour les touristes français avec ce « rêve oriental » ? Comment le marché doit-il réagir pour s'adapter aux attentes de la clientèle d'aujourd'hui qui tend à la diversité, la personnalité et la participation? Ces questions nécessitent des recherches et des études approfondies pour suivre et accompagner le developpement de cette destination touristique multiple qu'est la Chine.

\section{NOTES}

${ }^{1}$ Cette recherche est subventionnée par le projet de recherche des sciences humaines et sociales du Ministère de l'Éducation de Chine (20YJC740022).

${ }^{2}$ Source : Ministère de la Culture et du Tourisme de la République populaire de Chine, 2019. Il s'agit des chiffres globaux qui incluent les visiteurs français de tous les motifs, mais la présente étude vise avant tout le tourisme de loisir.

${ }^{3}$ L'enquête auprès des touristes est un peu plus ancienne que notre enquête auprès des TO puisqu'elle s'est déroulée dans le cadre d'une thèse défendue en 2012 ; mais les professionnels ont confirmé qu'il y avait peu de changements ces dix dernières années au niveau des activités touristiques proposées aux touristes français. Elle a été effectuée sur la base d'un questionnaire pendant 23 jours au mois d'août 2009 dans le centre de Yangshuo, principalement dans la rue de l'Ouest et quelques petites rues parallèles avec des hôtels et des restaurants du quartier où nous rencontrions facilement des touristes français. Notre échantillon est constitué de manière aléatoire par des touristes de nationalité française, âgés de plus de 15 ans, qui habitent en France métropolitaine et viennent en Chine pour motif touristique.

${ }^{4}$ Yangshuo et Guilin se classent devant des métropoles telles que Pékin, Shanghai et Canton, car c'est la région où cette enquête s'est déroulée. En général, les trois derniers sont les principaux lieux d'accueil des arrivées internationales. 


\section{BIBLIOGRAPHIE}

Axiamujiang, M., Hiyane, A. \& Yamamoto, K. (2016). Japanese Tourists' Consciousness of Foreign Travel: Towards Tourism Promotion of the Xinjiang Uygur Autonomous Region, China. Journal of the Japanese Forest Society, 98(2), 74-78.

Bai, C. (2005). Le développement touristique de la rue de l'Ouest à Yangshuo, Guilin : raisons, changements et tendances. Mémoire de Master, Nanning, Université du Guangxi, 76 p.

Boyer, M. (1996). L'invention du tourisme. Paris : Éditions Gallimard, Coll. Découvertes, 160 p.

Cuvelier, P. (1998). Anciennes et nouvelles formes de tourisme, une approche socio-économique. Paris : L'Harmattan, Coll. Tourismes et sociétés, $238 \mathrm{p}$.

Équipe MIT. (2002). Tourisme 1 : Lieux communs. Paris : Belin, $320 \mathrm{p}$.

Équipe MIT, (2005). Tourisme 2 : Moments de lieux. Paris : Belin, $352 \mathrm{p}$.

Équipe MIT, (2011). Tourismes 3 : Révolution durable. Paris : Belin, 332 p.

Jullien, F. (1991). Éloge de la fadeur, à partir de la pensée et de l'esthétique de la Chine. Paris : Editions Philippe Picquier, 142 p.

Mondou, V. \& Violier, P. (2009). Projets, pratiques et lieux touristiques, quelles relations? Mappemonde, 1-15.

Nyiri, P. (2005). Scenic spots: Chinese tourism, the state, and cultural authority. Seattle: University of Washington Press, 143 p.

Office national du Tourisme de Chine à Paris, Liste non exhaustive des agences de voyage françaises programmant la Chine, URL : http://www.otchine.com/ Agences de Voyage.htm, consulté le 29 mai 2020.

Qu, Y., Qu, H. \& Chen, G. (2016). Market segmentation for a leverage revitalization of China's inbound tourism: the case of US leisure tourists. Current Issues in Tourism, 21(6), 646-662.

Santos, J. (1998). The role of tour operators' promotional material in the formation of destination image and consumer expectations: the case of the Peoples' Republic of China. Journal of Vacation Marketing, 4(3), 282-297.

Song, Z. (2006). Research on tourism development of contemporary China. Beijing: Economy and Management Publishing House, 312 p.
Tang, X. (2014). The Evolution of Chinese Tourism Development Policies (1949-2013): A Quantitative Perspective. Tourism Tribune, 29(8), 15-27.

Taunay, B. \& Violier, P. (2015). Un modèle chinois des pratiques touristiques? Analyse des spécificités et des invariants au niveau des pratiques et des lieux fréquentés par les touristes chinois et internationaux en Chine. In I. Sacareau, B. Taunay \& E. Peyvel (Eds.). La mondialisation du tourisme, Les nouvelles frontières d'une pratique. Presse Universitaire de Rennes, Espaces et Territoires, 95-112.

Taunay, B. (2009). Le tourisme intérieur chinois : approche géographique à partir de provinces $d u$ Sud-Ouest de la Chine. Thèse de géographie, La Rochelle, Université de la Rochelle, 471 p.

Tomigová, K., Mendes, J. \& Pereira, L. (2016). The Attractiveness of Portugal as a Tourist Destination: The Perspective of Czech Tour Operators, Journal of Travel \& Tourism Marketing, 33(2), 197-210.

Urry, J. (1990). The tourist gaze: leisure and travel in contemporary societies. London: Sage Publications, $180 \mathrm{p}$.

Villard, F. (2006). China in French Tourist Industry Discourse: From Orientalist Imaginary to Chinese Postmodernity, Transtext(e)s Transcultures, 138-153.

Violier, P. (2000). Points de vue et lieux touristiques du monde. Mappemonde, 57(1), 7-11.

Violier, P. (2011). Les lieux du monde. EspacesTemps. net, URL: https://www.espacestemps.net/en/articles/ les-lieux-du-monde/.

$\mathrm{Xu}, \mathrm{F}$. (2004). The panorama on tourism marketing in China. Beijing: China Travel and Tourism Press, $328 \mathrm{p}$.

$\mathrm{Xu}, \mathrm{Z}$. (2003). Analysis on the Characteristics and Development Trend of Inbound Tourism in China. World Regional Studies, 12(3), 98-105.

\section{Coordonnées de l'auteur :}

Lu LI

Maître de Conférences

Université Sun Yat-sen (Chine) lilu@mail.sysu.edu.cn 
